\title{
PC And Speech Recognition Based Electrical Device Control
}

\author{
Sanchit Dua \\ IT LIMAT, Faridabad \\ India \\ Sanchit_dua@yahoo.com, sanchit_dua@in.com
}

\begin{abstract}
PC. It consist a circuit for using the printer port of a PC, for control application using software and some interface hardware. The interface circuit along with the given software can be used with the printer port of any PC for controlling up to eight equipments. Parallel port is a simple and inexpensive tool for building computer controlled devices and projects. The simplicity and ease of programming makes parallel port popular.
\end{abstract}

\section{Index terms: Electronic device controller, Diode, Transformer}

\section{INTRODUCTION}

We are controlling electrical devices connected with PC parallel port, either by wired link or wireless (optional) link. The output from parallel port of PC is TTL i.e. 5V. It is not advisible to drive the load relay directly to the PC parallel port as the current at this point is quite low. Thus we have to connect a relay driver circuit with this.

The software in VB or .Net will access the input from the microphone and the devices connected to the parallel port will get operated with this.

The software initially get it trained to recognize a particular word say 'one' or 'two', the project according to this will sense the mic. Input provided and the device corresponding to that voice will get activated from the parallel port output.

The relay driver unit consist of a regulated power supply and a single transistor operated simple relay switching circuit. When the TTL logic 1 comes from PC, the transistor gets activated and the device connected to the relay gets 'on' and vice versa. The software controlling the whole circuit, working on the real time of PC provides the desired output a per one's personal choice of time.

We are controlling electrical devices connected with PC parallel port, either by wired link.

\section{PROBLEM FORMULATION}

A. To design four circuits for relay driver Unit using transistor as switch.

B. To design a regulated power supply compatibles for the above relay driver circuit using suitable voltage regulator.
C. To write a program in VB/.NET (any one) etc. using GUI for parallel port interfacing for electrical device switching circuit with real time operating.

D. To design an IR/RF Transmitter/Receiver Circuit for the devices connectivity to access with remote operation (Load Connected at distant Location).

E. To attach the multimedia headphone with the audio adaptor card for audio input to be provided using microphone.

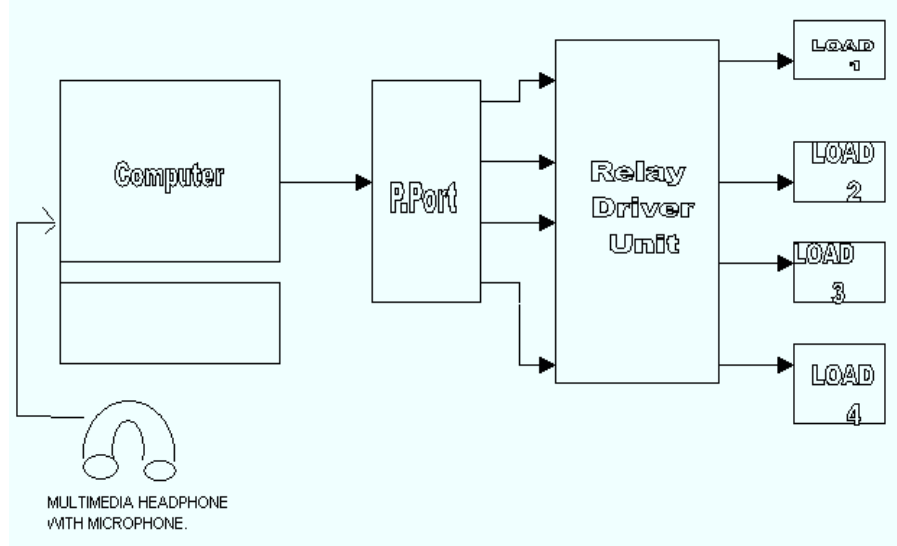

Fig 1. Wired Link

III. PARALLEL PORT

Hardware:-The pin outs of DB25 connector is shown in the picture below:

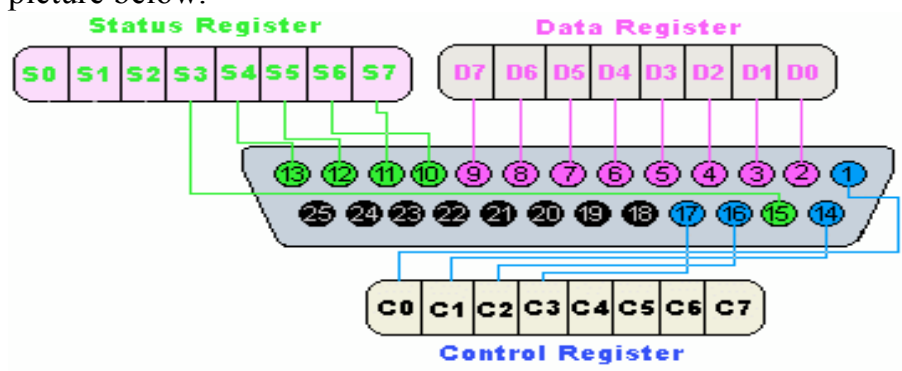

Fig 2. The pin outs of DB25 connector

The lines in DB25 connector are divided into three groups, they are:

A. Data lines (data bus)

B. Control lines 


\section{Status lines.}

As the name refers, data is transferred over data lines. Control lines are used to control the peripheral, and of course, the peripheral returns status signals back to the computer through Status lines. These lines are connected to Data, Control and Status registers internally.

\section{A. Parallel port registers}

The Data, Control and Status lines are connected to there corresponding registers inside the computer. So, by manipulating these registers in program, one can easily read or write to parallel port with programming languages like ' $\mathrm{C}$ ' and BASIC.

The registers found in a standard parallel port are:
A. Data register
B. Status register
C. Control register

\section{RELAY}

A relay is an electrical switch that opens and closes under control of another electrical circuit. In the original form, the switch is operated by an electromagnet to open or close one or many sets of contacts. It was invented by Joseph Henry in 1835. Because a relay is able to control an output circuit of higher power than the input circuit, it can be considered, in a broad sense, to be a form of electrical amplifier.

These contacts can be either Normally Open (NO), Normally Closed (NC), or change-over contacts.

- Normally-open contacts connect the circuit when the relay is activated; the circuit is disconnected when the relay is inactive. It is also called Form A contact or "make" contact. Form A contact is ideal for applications that require to switch a high-current power source from a remote device.

- Normally-closed contacts disconnect the circuit when the relay is activated; the circuit is connected when the relay is inactive. It is also called Form B contact or "break" contact. Form B contact is ideal for applications that require the circuit to remain closed until the relay is activated.

- Change-over contacts control two circuits: one normally-open contact and one normally-closed contact with a common terminal. It is also called Form C contact.

\section{Operation}

When a current flows through the coil, the resulting magnetic field attracts an armature that is mechanically linked to a moving contact. The movement either makes or breaks a connection with a fixed contact. When the current to the coil is switched off, the armature is returned by a force that is half as strong as the magnetic force to its relaxed position. Usually this is a spring,but gravity is also used commonly in industrial motor starters. Relays are manufactured to operate quickly. In a low voltage application, this is to reduce noise. In a high voltage or high current application, this is to reduce arcing.

If the coil is energized with DC, a diode is frequently installed across the coil, to dissipate the energy from the collapsing magnetic field at deactivation, which would otherwise generate a spike of voltage and might cause damage to circuit components. If the coil is designed to be energized with AC, a small copper ring can be crimped to the end of the solenoid. This "shading ring" creates a small out-of-phase current, which increases the minimum pull on the armature during the AC cycle.

By analogy with the functions of the original electromagnetic device, a solid-state relay is made with a thyristor or other solid-state switching device. To achieve electrical isolation, a light-emitting diode (LED) is used with a photo transistor.

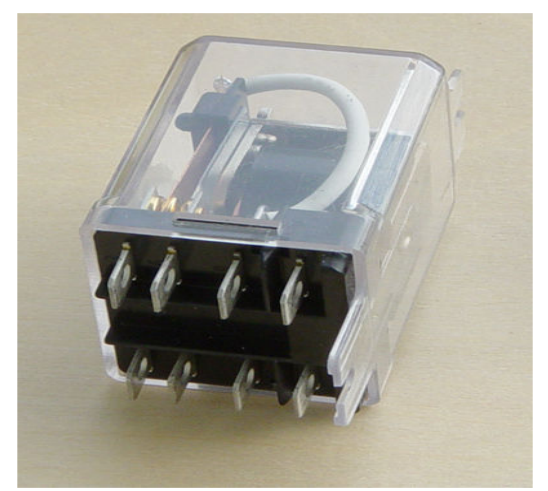

Fig 3. RELAY 
Fig 4. Circuit Diagram Of Project

\section{CAPACITORS}

It is an electronic component whose function is to accumulate charges and then release it.

To understand the concept of capacitance, consider a pair of metal plates which all are placed near to each other without touching. If a battery is connected to these plates the positive pole to one and the negative pole to the other, electrons from the battery will be attracted from the plate connected to the positive terminal of the battery. If the battery is then disconnected, one plate will be left with an excess of electrons, the other with a shortage, and a potential or voltage difference will exists between them. These plates will be acting as capacitors. Capacitors are of two types: - (1) fixed type like ceramic, polyester, electrolytic capacitors-these names refer to the material they are made of aluminium foil. (2) Variable type like gang condenser in radio or trimmer. In fixed type capacitors, it has two leads and its value is written over its body and variable type has three leads. Unit of measurement of a capacitor is farad denoted by the symbol F. It is a very big unit of capacitance. Small unit capacitor are pico-farad denoted by $\mathrm{pf}(\mathrm{Ipf}=1 / 1000,000,000,000 \mathrm{f})$ Above all, in case of electrolytic capacitors, it's two terminal are marked as (-) and $(+)$ so check it while using capacitors in the circuit in right direction. Mistake can destroy the capacitor or entire circuit in operational.

\section{DIODE}

The simplest semiconductor device is made up of a sandwich of P-type semiconducting material, with contacts provided to connect the p-and n-type layers to an external circuit. This is a junction Diode. If the positive terminal of the battery is connected to the p-type material (cathode) and the negative terminal to the N-type material (Anode), a large current will flow. This is called forward current or forward biased.

If the connections are reversed, a very little current will flow. This is because under this condition, the p-type material will accept the electrons from the negative terminal of the battery and the N-type material will give up its free electrons to the battery, resulting in the state of electrical equilibrium since the N-type material has no more electrons. Thus there will be a small current to flow and the diode is called Reverse biased.

Thus the Diode allows direct current to pass only in one direction while blocking it in the other direction. Power diodes are used in concerting AC into DC. In this, current will flow freely during the first half cycle (forward biased) and practically not at all during the other half cycle (reverse biased). This makes the diode an effective rectifier, which convert ac into pulsating dc. Signal diodes are used in radio circuits for detection. Zener diodes are used in the circuit to control the voltage.

\section{A. Principle of Transformer}

Two coils are wound over a Core such that they are magnetically coupled. The two coils are known as the primary and secondary windings.

In a Transformer, an iron core is used. The coupling between the coils is source of making a path for the magnetic flux to link both the coils. A core as in fig.5 is used and the coils are wound on the limbs of the core. Because of high permeability of iron, the flux path for the flux is only in the iron and hence the flux links both windings. Hence there is very little 'leakage flux'. This term leakage flux denotes the part of the flux, which does not link both the coils, i.e., when coupling is not perfect. In the high frequency transformers, ferrite core is used. The transformers may be step-up, stepdown, frequency matching, sound output, amplifier driver etc. The basic principles of all the transformers are same.

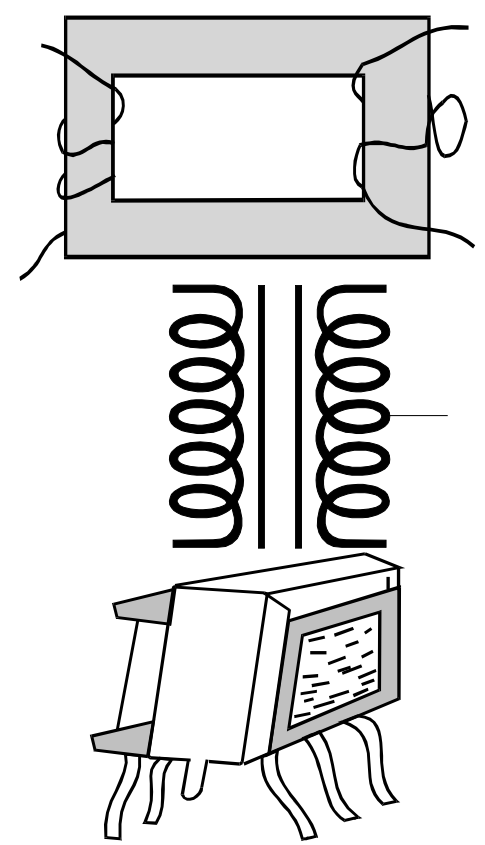

Fig 5. Transformer Equipments

\section{SPEECH RECOGNITION}

A. Introduction

Speech Recognition(also known as automatic speech recognition or computerspeech recognition) converts spoken words to machine readable input(for ex, to key presses, using the binary code for a string of characters codes).Tthe term "voice recognition" is sometimes used to referto speech recognition where the recognition

System is trained to a particular speaker, as is the case for most desktop recognition software, hence there is an aspect of speaker recognition, which attemts to identify the person speaking, to better recognize what is being said.Speech 
recognition is a broad term which means it can recognize almost anybody's speech such as a call centre system designed to recognize many voices. Voice recognition is a system trained to a particular user, where it recognizes their speech based on their unique vocal sound.

1) Applications

a) Heath Care

b) Military

c) Telephony

d) People with disabilities

e) Further applications

IX. List of Instruments and Equipment needed for the project work

\section{A. Hardware}

- $\quad$ One computer with $1 \mathrm{~GB}$ of memory

- $20 \mathrm{~GB}$ hard disk space

- An Intel Pentium Core 2 Duo based computer working at least @ $2.2 \mathrm{GHz}$ speed

- 15 inch monitor

- A microphone

- $\quad$ Transmitter and Receiver

- $\quad$ Relay driver unit

- $\quad$ Parallel Port

- Electrical Devices

\section{B. Software}

- $\quad$ Programming Languages

- $\quad$ Speech Recognition Software

- Readymade application software developed by scholars or institutions.

\section{REFERENCES}

[1] Expandable home automation systems by launey, February, 1992.

[2] Humphrie, Home automation system, Int.J. of Adv. Computing, Vol. 5, Issue 6, April, 1997 .

[3] Access control apparatus for use with buildings, gated properties and the like by Parsadayan, March, 2000.

[4] Using speech recognition to access the Internet, including access via a telephone by Scott, August, 2000.
[5] Speech Recognition system for electronic switches in a non-wireline communications, Network by Baries, December, 2000 .

[6] Speech Recognition front end controller to voice mail systems by O'Brien, July, 2003.

[7] System and method for monitoring and controlling residential devices by Petite, May, 2005.

[8] Voice control system for operating home electrical appliancesby Kawasaki , January, 2006.

[9] Use of a PC Printer Port for Control and Data Acquisition by Peter H. Anderson.

[10] What you need to know about electronics telecards by Stephane Bausson.

[11] Your PC's Printer PORT: A Window on the real World by Tom Dickinson.

[12] www.searchableonline-bibiliography.com for Phinetics and Speech technology with more than 8000 entries.Provided by Institut fur phonetic at Johnn Wolfgang Goethe-UniversityFrankfurt.

[13] [Z]L. R.Rabiner and B.H.Juang, Fundamentals of Speech Recognition,New Jersey,Prentice-Hall,Inc, 1993.

[14] S.Euler and J.Zinke,"The influence of speech coding algorithms on automatic speech recognition" in Proc.1994 Int. Conf.Acoustics, Speech and Signal Processing, Adelaide, Australia, 1994.

[15] L.R.Rabiner and B.-H Juang.Fundamentals of Speech Recognition Englewood Cliffs, NJ:Prentice-Hall, 1993.

[16] A Potamianos and V.Weerackody,"Soft-feature decoding for Speech recognition over wireless channels" presented at the Int.Conf.Acoust.Speech and Signal Processing, Salt Lake City, UT, Mat, 2001.

[17] L.A.Bryan, E.A.Bryan, Programmable Controllers, Industrial Text Co, Chicago, IL, 1988. 